\title{
EAI Endorsed Transactions

\section{The Effect of Congestion Control Model on Congested Traffic Flow in Vehicular Ad Hoc Networks (VANETs)}

\author{
Adams A.K. Azameti ${ }^{1,}{ }^{*}$, Ferdinand A. Katsriku ${ }^{2}$, Peter Chong ${ }^{3}$ and Jamal D. Abdulai ${ }^{4}$ \\ ${ }^{1} 1$ st University of Ghana, Computer Science Dept. aakazameti@st.ug.edu.gh \\ ${ }^{2} 2$ nd University of Ghana, Computer Science Dept., fkatsriku@ug.edu.gh \\ ${ }^{3} 3$ rd Auckland University of Technology, Electrical \& Electronic Eng. Dept., peter.chong@aut.ac.nz \\ ${ }^{4} 4$ nd University of Ghana, Computer Science Dept., jabdul@ug.edu.gh
}

\section{Abstract}

\begin{abstract}
This study seeks to conduct analytical investigation into Congestion Control models to determine the effects of parameters inherent in mitigating traffic flow density in a congested network. The spatial temporal variations in the value of $\lambda$ affects vehicular traffic flow. This study proposed a Congestion Control Model and perform simulation in MATLAB. In this model, vehicular density and number of vehicles were held constant while varying the value of $\lambda$ from 1 to 10 . The simulation result reveals the vehicle trajectories as the value of $\lambda$ increases with time. The outcome of this study indicates that there is no need increasing the value of $\lambda$ beyond 12 , since further increases would only increase the number of vehicles leading to traffic jam. In principle, it is very important to use $1 \leq \lambda \leq 12$ in designing traffic models in VANETs The analytical result proved that the Congestion Control Model for the four-lane may be the most appropriate model to be adopted and use in designing VANETs networks.
\end{abstract}

Keywords: Ad Hoc Networks, Mobile Ad Hoc Networks, Sensor Networks, Vehicular Networks.

Received on 30 October 2017, accepted on 12 November 2017, published on 22 March 2018

Copyright $\odot 2018$ Adams A.K. Azameti et al., licensed to EAI. This is an open access article distributed under the terms of the Creative Commons Attribution licence (http://creativecommons.org/licenses/by/3.0/), which permits unlimited use, distribution and reproduction in any medium so long as the original work is properly cited.

doi: 10.4108/eai.22-3-2018.154371

\section{Introduction}

In recent times, there has been a great deal of research efforts from both academia and industry to revolutionize the world of transportation [1,2]. This effort has been directed at building Intelligent Transportation Systems (ITS) with the aim of improving road safety as well as traffic efficiency. This has been made possible because of advances in hardware, software and communication technologies. The successful implementation of ITS will lead to greater driver and passage comfort. Under the auspices of the Electronic Safety Initiative (i.e. GST,Pre Vent etc) [3]. The European Union nations have taken the lead to investigate into the future potential of reducing road fatalities through the use of vehicle-to-vehicle (V2V) communication. Much of the research in this area has been directed at finding novel design architectures, routing protocols, security as well as quality of service. The leaders in this effort has been the European Union, USA and Japan where there have been trials and deployments.
The Car-to-Car communication (C2CC), usually referred to as vehicle-to-vehicle (V2V) communication in the vehicular ad hoc network (VANETs), has the potential to enable many new applications for vehicles. This would eventually create numerous future opportunities for enhanced safety improvements in vehicular ad hoc networks. The communication between vehicles has the potential to support drivers and active safety applications such as collision warning, up-to date traffic and weather information and navigation systems.

However, the future application of these services in VANETs, pose numerous challenges with the technology, protocol designs, security and this has become an active research area that has attracted the attention of both industry and academia globally.

Inter-Vehicle Communication (IVC) Systems in VANETs is seen to attract considerable attention from the research community and automotive industry. This event is being spearheaded by many automobile manufacturers and have step-up efforts to build communication devices for future

*Azameti: adamsblessed7@gmail.com 
vehicles to ensure safety, comfortable driving, and entertainment. Each year more than six million crashes in the U.S. cause almost thousands of deaths and millions of injuries and other countries are not excluded either.

The vehicle-to-vehicle and vehicle-to-infrastructure communication can prevent some of these collisions once it is developed. This occurs by warning drivers or on-board computers in vehicles about dangerous situations such as accidents, slippery road segments, stopping traffic and other vehicles approaching intersections. In addition to preventing accidents, communication among vehicles can increase the efficiency of the transportation systems.

VANETs have almost the same characteristics as mobile ad hoc networks in the form of multi-hop networks communications. Due to the mobility patterns of the nodes coupled with dynamic network topology changes, all the nodes utilize the same channel leading to traffic congestion in dense network.

The decentralized capability in VANETs leads to the need for a paradigm shift for new system concepts and V2V communication. Besides, the decision on the wireless communication standard to be used and message dissemination schemes capable of exchanging messages under different networks scenarios. This advancement, will improve high speed real-time communication to enable collision warning.

The mobility in the Vehicular Ad hoc Networks (VANETs) are usually considered as decentralized and selforganizing networks that are made up of high speed moving vehicle. This unique feature of VANETs is used to establish communication among vehicles and therefore develop pervasive application for road safety.

However, the acceptable communication mode in VANETs is through broadcast and these characteristics of VANETs is the major challenge. This undoubtedly renders emergency traffic alert message dissemination very difficult due to the high mobility and fast topology changes in vehicular environments. Due to this phenomenon in VANETs, it is important to substantiate the position of the farthest vehicles (nodes) through different methodology.

For us to accomplish this in vehicular Ad hoc networks, it is of utmost important to use decentralized controllers to predetermine the exact threshold parameters.

This will eventually help vehicles (nodes) to dynamically decide on the appropriate timelines in which the rebroadcast should be sent and when to discard received emergency traffic alert. This study seeks to conduct analytical investigation using macroscopic traffic flow models to ascertain the inherent effects of $\lambda$ as a parameter in congested networks.

\section{Related Works}

Data dissemination in vehicular networks is primarily concerned with the delivery of safety information in a timely manner such as accident warnings to drivers to enable them take corrective measures to avoid fatalities on the roads. The most appropriate packet delivery mechanism required to support such applications is usually multicast in nature. However, the membership of the multicast group is defined implicitly by driving direction, location, speed and time, instead of explicit identification to ensure that all vehicles that are upstream of a traffic accident should be within one kilometre transmission range according to [4].

In [4], a system was proposed where the road is divided into short segments to enable the mobile or stationary nodes to aggregate the information relevant to the segment from sensors or received packets. This summary information is sent via one-hop broadcast to other nodes. In this case, the application layer is responsible for forwarding the persegment information further to other nodes after processing the relevant information. The nodes have the tendency to relate the information to the corresponding segments with the help of digital maps.

In urban environments, according to IEEE 802.11 based urban Multi-Hop Broadcast (UMB) protocol is proposed in [5]. This protocol is designed to assign the duty of forwarding and acknowledging broadcast packets to only one vehicle by dividing the road segment inside the transmission range into smaller sections and choosing the further non-empty section of the vehicle without giving any prior topology information such as the ID or position of its neighbours. In other circumstances, if an intersection lies in the path of the message dissemination, the authors propose the use of repeaters to initiate new directional broadcasts in all directions.

According to [6], the effect of vehicle mobility ensures that the message is stored while waiting for an opportunity to forward them for end-to-end delay to be reduced. $\mathrm{Bi}$ directional and multilane highways provide additional freedom of movement, which also reduces the delay to some extent. According to [7], four physical models are evaluated to measure the rate of information propagation. The paper examines the positive influence due to increases in operation rate, mobility rate and vehicle density, on the rapid propagation of information. In a typical urban environment, a hybrid physical model combines V2V and V2R communication models for enhanced performance.

\subsection{Applications}

A lot of applications were proposed within Intelligent Transportation Systems (ITS) projects in recent times. In advent of new advances in wireless technology and the research efforts from the academia and industry in mobile ad hoc and VANETs, the VANETs are seen with the capabilities of supporting these ITS applications.

\section{i. Safety Applications}

In VANET applications, vehicle is made intelligent to recognize that there is a dangerous situation ahead and has the capability of reporting it instantly to neighbouring vehicles. The hazardous situation can be identified by sensors to detect events such as the deployment of airbags due to accident, loss 
of tire attraction, or abrupt application of brakes. The critical requirements in these applications are latency and area of coverage as reported in [8].

The concept of Zone-of-Relevance was introduce in [9]. Within this zone, a warning message is delivered to drivers to assist them to avoid dangerous situations while the vehicle outside the Zone-of-Relevance may route the message but drivers are not alerted to ensure that only the relevant messages are delivered to drivers and all the unnecessary reactions are avoided to prevent fatality on the roads.

\section{ii. Traffic Monitoring}

The use of existing capabilities of vehicles, such as Car Area Network (CAN) and GPS, VANETs act as an intelligent sensor and forms powerful traffic information systems. The advantages of using VANETs over other infrastructure-based systems involved the rapid deployment, self-organization and lower-cost.

In the system proposed in [10], VANETs is composed of fixed and mobile nodes (vehicles). The vehicles are organized into clusters where they exchange beacon signals to maintain a neighbouring relationship. This task requests are generated by nodes or from an external network and are transmitted to immediate neighbours to monitor some relevant conditions of the roads. During the process of forwarding the tasks requests may occur to propagate the requests through the network and upon receiving the request, the vehicle samples the data from the relevant sensor. This data is then summarized and forwarded back to the originating nodes as a task response.

According to these authors [4], each vehicle monitors the locally observed traffic situation by receiving data packets concurrently with detailed information from other vehicles. In their studies, a traffic situation analysis is performed in each individual vehicle and the result is transmitted through a wireless data-link to all vehicles in the local neighbourhood.

\section{iii. Cooperative Driving in Automated Highways}

This types of VANETs applications are primarily concerned with the automation of certain driving functions to expedite efforts toward driving safely and improve the capacity of highways. This study investigates platoon formation and lane merge according to $[11,12]$ and obstacle avoidance and blind intersection assistance as indicated in $[13,14]$.

\section{iv. Entertainment}

These applications ensure the delivery of multimedia content for vehicle passengers. Among the challenges in this area is the provision of QoS guarantees and locating the media content. The author in [15], assess the importance of a Car-to-Car (C2C) and Peer-to-Peer (P2P) systems for delivering media content using the cellular and the vehicular ad hoc networks (VANETs).

\subsection{The Vehicular Mobility}

Many researchers are highly interested in studying sparse vehicular networks due to its importance in ITS applications in VANETs. This motivation from most researchers reflects the assumptions inherent in the inter-vehicle communication in sparse networks as more challenging due to the difficulty in maintaining connectivity. In addition, the low-density conditions offer more flexibility in setting the traffic parameters such as flow, speed and density for analytical and simulation studies. The assumption of independent parameter is valid only in free-flow traffic. Therefore, the results of these studies cannot be used directly in dense traffic environments. This study seeks to argue that research should focus more on dense traffic and traffic jam environments due to their important significant influence on VANETs, since:

i. The vehicle density can contribute to rapid message dissemination [16-19] .

ii. Higher node density aggravates the contention and collision problem in the communication protocols based on shared media.

iii. The traffic jams contribute to certain nonhomogeneous distribution of vehicles and therefore increasing the range of transmission needed to maintain connectivity.

iv. The vehicle traffic may change rapidly between free-flow in congested traffic as the result of the traffic controls, road constraints or accidents.

The review of research directions related to the use of vehicle mobility models and traffic simulator is provided in the next subsection.

\subsubsection{The Vehicle Mobility Models and Traffic Simulations in VANETs}

Vehicular mobility models and traffic simulators have become popular for use in VANETs studies [20]. The main reasons for these simulators is for use in traffic analysis, forecasting and planning transportation networks. Due to the important applications in VANETs, the ITS initiative has generated an enormous number of vehicular traffic simulators since its inceptions in the United State in 1991 according to [21]. These simulators are intended to support ITS applications, but not necessarily VANETs due to their recent introduction.

VANETs research papers, simulation for vehicle movement is usually considered a special type mobility model according to $[8,22]$. These mobility models cannot produce complex vehicle manoeuvres while car-following, decelerating, and accelerating, during traffic jam situations. For the analysis of any complex phenomenon like VANETs, 
many studies have relied on vehicular mobility models to generate vehicle movement traces.

\section{i. The Mobility Models}

Many studies in ad hoc networks often require keeping track of nodes positions at any given time and vehicular networks are no exception. In recent time, many VANETs applications depend on realistic vehicle movement patterns and this can be obtained from traffic micro-controllers which is capable of tracking the characteristics of individual vehicles including the position, speed and direction. The early research in inter-vehicle communication relied on microsimulators that were designed for the purpose of investigating vehicle traffic in transportation[23] on networks. VANETs research adapts simulators to generate traces of vehicles positions at regular time interval into network simulator such as ns-2, ns-3, MATLAB as outlined in these papers [18, 23$26]$ to perform simulation of communication protocols. In recent times, the research communities have devoted substantiate effort to the development of network traffic simulators for the purpose of VANETs research as reported in $[18,23]$. Usually, these simulators have a dual focus on the communication and mobility sides of VANETs.

\section{ii. The Traffic Flow Models}

The traffic simulators are extensively used in evaluating vehicular ad hoc networks. The analytical approaches in freeflow traffic conditions are usually the preferred choice. The primary reasons for this choice is the control environments it offers, ability to select at least two or three parameters to establish the fundamental relationship of the traffic flow. Under free-flow conditions, according to [27-30], it is assumed that the traffic flow and the vehicle velocity are independent. This fundamental relationship of traffic flow is used to derive probability density function (pdf) of the communication duration between vehicles and the pdf of the time between consecutive topology changes. Similar assumptions are made in [31] to derive the pdf for distance at which to establish a multi-hop connection between two vehicles. The effective selection of time headway (i.e. the time gap between consecutive vehicles) as an input parameter can be control both the rate by which vehicles are injected to the simulation environment and the density of the vehicles. According to [32], the time headway values are generated randomly from a Poisson distribution with an average value that ensures the free-flow traffic. A uniform distribution with a minimum value that specifies the initial separation between vehicles is used in [31].

\section{iii. The Vehicle Speed}

In most cases, the desired speed is provided as an input to the simulations. The vehicle speed can be a constant value for each type of vehicles (e.g. cars and trucks) or may be sampled from a random distribution. In [33], the speed distribution is chosen to correspond to real measurements from German highways, while in $[5,13]$, the speed is assigned from a normal distribution.

One possible choice of random vehicle speed is based on the theory of the " $85^{\text {th }}$ percentile speed" that is usually used as a guide to set limit on the highways as reported in [34-37]. The $85^{\text {th }}$ percentile speed is defined as the speed $85 \%$ of drivers moving at or below. It is evident that $75 \%$ of the drivers maintain a speed within a certain range around the average speed limit. Another research findings indicates that $15 \%$ are slow drivers while the remaining $15 \%$ are driving at high speeds. According to $[6,28]$, vehicle speed is sampled from a Normal distribution using the standard deviation to mark the boundaries between slow, average and high speeds. Although, the speed and time headway changes in real traffic, they are kept constant in [38-41].

Furthermore, the choice of the average speed varies according to the choice of the environment under considerations. For instance, in highway simulations environment the average speed is set to $100-130 \mathrm{~km} / \mathrm{hr}$ according to [27, 33]. Similarly, the simulations of city street grid set the average speed to $40-80 \mathrm{~km} / \mathrm{hr}$ either randomly according to [5] or according to the street types [42]. The choice of speed may reflect also the posted speed limit, road conditions and common driving habits.

\section{iv. The Collisions}

The collision in VANETs is usually assumed in some literatures that vehicles moves freely and collisions are not allowed. However, the vehicle density according to [32] is specified to create congested traffic situations and vehicles may collide if drivers fail to react in time. This finding is very necessary to evaluate the influence of the V2V and V2R communication on safety as indicated in [32].

\section{v. The Road Topology}

This may be described as a common choice of highway geometry of two or more lanes in each direction. This allows the traffic to flow in both directions may be described as asymmetric as indicated in [27]. According to [11], they argue that dividing the highways may be used to limit the geographic area affected by message broadcast to one traffic direction. According to [42-44], explained more complex geometry to be used to evaluate routing strategies and protocols in a city traffic flow environment.

This paper is organized as follows. Section 2, discusses the state-of-the-art related work. The proposed Congestion Control Model (CCM) is provided in section 3; the performance evaluation and simulation results are presented in section 4. Finally, draw a conclusion and outlined future research direction in Section 5. 


\section{Proposed Traffic Models}

This section introduces the proposed Congestion Control Models (CCM) for four-lane road networks. The Model seeks to take into account the entire aggregation of vehicles traffic flow states and its inherent density disruptions as catalyst to mitigate congestion in VANETs. In fact, these density disruptions to some extent are due to the correlations between parameters such as speed, density and vehicle mobility pattern.

The proposed Congestion Control Models (CCM) is intended to ascertain the general interactions of vehicular mobility pattern into congestion control. The congestion control seeks to mitigate traffic congestion by varying the value of $\lambda$. This macroscopic model is the main direction of this study according to [45]

\subsection{A Congestion Control Models (CCM)}

This model describes entities, their activities and interactions at low level of detail. For example, a traffic stream may be represented in the form of a scalar value of flow, density and speed. This model ensures that the traffic stream is properly allocated to lane rather than lane-changes. This model deal with the aggregate behavior of the traffic flow states.

Another classification addresses the process represented by the model as either deterministic or stochastic. The deterministic models define all the entities with their interactions by the exact relationships using mathematical, statistical or logical for its representations. The stochastic models have processes with probability functions. A carfollowing model, for instance, may be formulated either as a deterministic or stochastic relationship by defining the driver's reaction time as a constant value or a random variable respectively. In this proposed model, the study adopts the well-known classifications of macroscopic traffic flow for different densities with particular focus on the congested network as shown in table. 1

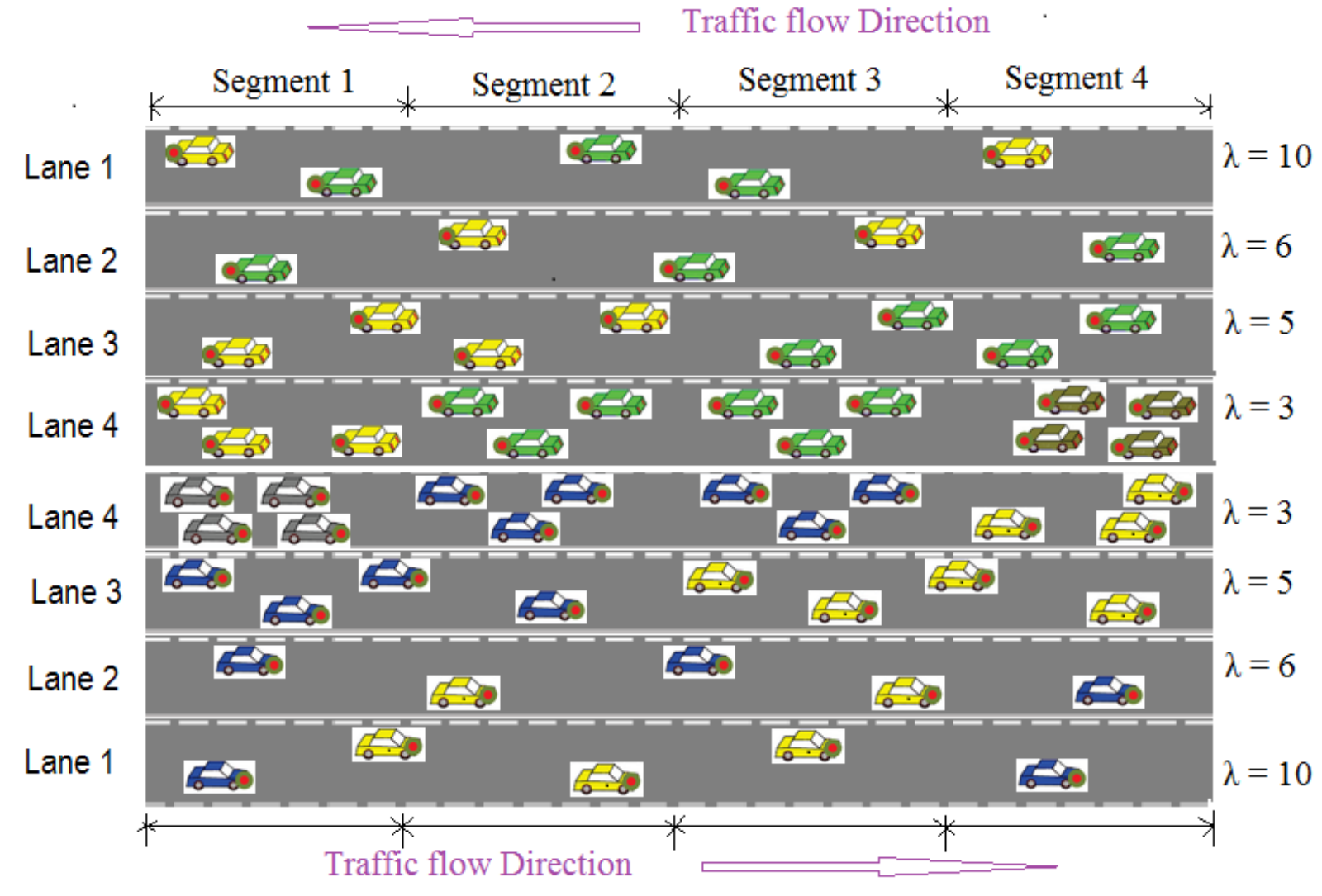




\subsection{Low Traffic Design}

The vehicles under low traffic density and their interaction between vehicles are very low and therefore experiences free traffic flow movement. This free flow condition, results in realistic independent movement among vehicles without any restriction on the roads. The computational model developed in [45-48], indicates that the arrival process of vehicles has a highway density. This can be modelled as a Poisson process with an arrival rate $\lambda_{t}$. Therefore, the probability that $n$ vehicles arriving in a time period t can be computed using. The equations are shown in number (1), (2) and (3).

$$
P\left(X=n \quad \frac{(x \lambda t)^{n}}{n !} e^{-\lambda t x}\right.
$$

$\chi$, is a number of vehicles migrating to a highway in a time interval $x$. The time headway distribution is described in $[46,49]$, as an exponential distribution of this parameter $\left(\frac{1}{\lambda_{t}}\right)$ with the probability density function (PDF) $f T(t)=\lambda_{t} e^{-\lambda t \chi}, t \geq 0$. The PDF allows the time headway to be zero and this actually give it the highest relative likelihood of occurrence which is not possible to be zero by the set conditions. For a realistic result to be achieved, it is important to increment with the minimum allowable time headway. This PDF is given by

$$
f_{T}()=\left\{\begin{array}{l}
\lambda t e^{-\lambda t(t-\tau),} t \quad \tau \\
0, \quad t<\tau
\end{array}\right\}
$$

$$
D=\frac{V}{v}
$$

Where $V$ in equation (3) is the traffic volume me a sured in vehicle per hour (veh/hr) and D, density is measured in vehicle per mile per lane in (veh/mile/lane), and $r$ epresents the space mean speed in mph. The traffic

\begin{tabular}{|c|c|c|c|}
\hline $\begin{array}{l}\text { Density } \\
\text { (Veh/Mile/Lane) }\end{array}$ & $\begin{array}{l}\text { Level of } \\
\text { Service }\end{array}$ & Flow conditions & \\
\hline $0-12$ & $\bar{A}$ & Free-flow operations & \multirow{4}{*}{$\begin{array}{l}\text { Uncongested flow } \\
\text { conditions }\end{array}$} \\
\hline $12-20$ & $\bar{B}$ & Reasonable free-flow operations & \\
\hline $20-30$ & $\mathrm{C}$ & Stable operations & \\
\hline $30-42$ & $\mathrm{D}$ & Borders on unstable operations & \\
\hline $42-67$ & $\mathrm{E}$ & \begin{tabular}{|lll} 
Extremely & unstable & flow \\
operations & & \\
\end{tabular} & $\begin{array}{l}\text { Near-capacity } \\
\text { flow conditions }\end{array}$ \\
\hline $67-100$ & \multirow[t]{2}{*}{$\overline{\mathbf{F}}$} & Forced or breakdown operations & \multirow[t]{2}{*}{$\begin{array}{l}\text { Congested flow } \\
\text { conditions }\end{array}$} \\
\hline$>100$ & & Incident situation operations & \\
\hline
\end{tabular}
flow conditions corresponding to each density level are classified in Table 1. [45]

Table 1. Traffic flow state for different densities [45]

\section{The Performance Evaluation}

This section outlines the step-step approach to carry-out a comprehensive analysis to arrive at the desired results.

\subsection{The Simulator}

This study evaluates the performance of the macroscopic traffic models based on the event driven simulation [50-52]. 
The mobility of the vehicle and the road network is being simulated using MATLAB with system parameters in the table. 2 below.

\begin{tabular}{|c|l|l|}
\hline Item Number & Parameters & Descriptions \\
\hline 1 & Platform & MATLAB \\
\hline 2 & Speed of Vehicles & $60 \mathrm{~km} / \mathrm{hr}$ \\
\hline 3 & No. of Vehicles on the Road & 1800 \\
\hline 4 & Type of Road (Capacity) & Highways, Urban, Semi-Urban \\
\hline 5 & Number of Lanes in each direction & 4 \\
\hline 6 & Average Percentage of vehicles & $30 \%$ \\
\hline 7 & Expected Threshold Changes & $15 \%$ \\
\hline 8 & Speed Limit & $36 \mathrm{mile} / \mathrm{hr}$ \\
\hline
\end{tabular}

Table 2. System Parameters

\subsection{The Four-Lane Traffic Mobility}

This study modifies the known civil engineering traffic flow models in Four-Lane Mobility in VANETs within a congested network. This study demonstrates how the network behaves under varying traffic densities which involves 10Miles $\mathrm{veh} / \mathrm{mile} / \mathrm{lane}$ to $100 \mathrm{veh} / \mathrm{mile} / \mathrm{lane}$ within $15 \mathrm{Miles} \mathrm{road}$ section to investigate network behaviour with four lanes analytically.

To really understand the interactions among two vehicles in terms of traffic flow density. This study seeks to vary the value of lambda from 1 to 10 against $100 \mathrm{veh} / \mathrm{mile} /$ lane to investigate how the network behaves when $\lambda$ increases under in a congested network scenario.

The link layer contention delay statistics is measured with the central tendency indicating the various thresholds of all vehicles receiving the broadcast packet during the broadcast storm at different probability densities as clearly shown in Fig 1-10.

\subsection{The Performance Metrics}

This $\lambda$, is an important parameter used to model the behaviour and the interaction of vehicles at different traffic densities. The study used $67 \mathrm{veh} / \mathrm{mile} / \mathrm{lane}$ to $100 \mathrm{veh} / \mathrm{mile} / \mathrm{lane}$ and varies the $\lambda$ from 1 to 10 to investigate the effects of $\lambda$ on the interaction of vehicles on the four-lane.

The network behaviour with respect to the lane changes are shown in the figure below If $\lambda=1$, the traffic flow trajectories within the four-lane exhibits exponential distribution where lane 1: has the least congestion with less priority in terms of packet reception. The next less priority occurs at lane 2 but has an increased number of vehicles than in lane 1. Lane 3, experiences high number of vehicles while lane 4 shows higher number of vehicles and therefore receives the highest priority reception rate to control the congestion. Lane 3, is the second higher lane with the next higher priority reception rate when lane 4 is reduced to the acceptable threshold as shown in

Fig. 3.

When $\lambda=2$, the traffic flow within the lanes also indicate an exponential distribution at lane 1 and lane 2 and therefore have least priority while lane 2 having fewer number of vehicles than in lane 1. Lane 3 and lane 4 begin to show partial normal distribution in Fig 4. Lane 4 has the highest priority in terms of packet reception and lane 3 has the next higher priority after lane 4 has successfully receives the needed reception.

Again, if $\lambda=3$, the traffic flow within the four-lane shows an exponential distribution at lane 1 and indicates a partial normal distribution from lane 3 and lane 4 in Fig 5. Since, lane 3 and lane 4 have partial normal distribution indicate that there is an increased number of vehicles but with lane 4 having the highest number of vehicles than in lane 3 . Therefore, lane 4 is congested with the highest priority reception rate and the next priority is lane 3 . When $\lambda=3$ shows the initial threshold in which lanes continue to experience an increased number of vehicles anytime the value of $\lambda$ is increased. 
When $\lambda=4$, the traffic flow shows a complete normal distribution at lane 3 and lane 4 with an increased number of vehicles as indicated in Fig 6. At this stage, lane 3 and lane 4 are congested but lane 4 has the highest number of vehicles and therefore need to receive the highest priority of reception rate before lane 3 . Lane 1 is still the least but with increased number of vehicles once the value $\lambda$, increases. Lane 1 , has high mobility than in lane 2 since the number of vehicles in lane 2 is higher than in lane 1 . Therefore, lane 1 has the highest mobility rate.

Subsequently, when $\lambda=5$, the traffic flow exhibits a complete normal distribution at lane 2, lane 3 and lane 4 as indicated in Fig 7. Lane 1 has the least number of vehicles and therefore has the highest mobility rate than in lane 2 . Lane 3 and lane 4 still have the highest number of vehicles, since both lanes become congested with lane 4 having the highest number of vehicles indicating a high priority in terms of packet reception and move subsequently to lane 3 .

In addition, when $\lambda=6$, the traffic flow also shows a complete normal distribution at lane 2, lane 3 and lane 4 as indicated in Fig 8. At this stage lane 3 and lane 4 have an increased number of vehicles and therefore congested but lane 4 is congested than lane 3 . Lane 4 , seems to experience higher congestion than lane 3 . Lane 1 , still has the least number of vehicles with reasonable amount of mobility rate than in lane 2 , lane 3 and lane 4 .

If $\lambda=7$, the traffic flow shows a complete normal distribution at lane 2, lane 3 and lane 4 as indicated in Fig 9. Lane 4 has the highest priority in terms of packet reception and move subsequently to next lane 3 . At this instance, is worth to note that anytime the value of $\lambda$ increases then all the four-lane also increases indicating a direct proportion in each lane.

When $\lambda=10$, the traffic flow shows a complete normal distribution for all the four-lane as indicated in Fig 12. Lane 4 has the highest priority in terms of packet reception and move subsequently to next lane 3 but with an increased number of vehicles. This trend shows that once all the lanes assumed a complete normal distribution it would correlate with an increased number of vehicles but with lane 3 and lane 4 term a congested network. In this instance, lane 4 has the highest packet reception next to lane 3 , lane 2 and lane 1 in that order of magnitude in terms of traffic density.

The spatial temporal variations in the value of $\lambda$ affects vehicular traffic flow. This study proposed a Congestion Control Model (CCM) and perform simulation in MATLAB. In this model, vehicular density and number of vehicles (veh/mile/lane) were held constant while varying the value of $\lambda$ from 1 to 10 . The simulation result reveals the vehicle trajectories as the value of $\lambda$ increases with time. The outcome of this study indicates that there is no need increasing the value of $\lambda$ beyond 12 , since further increases would only increase the number of vehicles (veh/mile/lane) leading to traffic jam. In principle, it is very important to use $1 \leq \lambda \leq 12$ in designing traffic models in VANETs. The analytical result proved that the Congestion Control Model (CCM) for the four-lane is first to none to be adopted and use in designing VANETs networks. In this model, each lane has a certain number of vehicles in a given period. First, lane 4 experiences congestion; follow by lane 3 ; next to lane 2 and lane 1 happens to experience free-flow of traffic.

This traffic flow phenomenon would help VANETs decentralised deployment of an autonomous vehicles capable of prioritising lane with the highest congestion is giving the minimum threshold to receive emergency traffic alert message while the rest of the lane remained waiting for the next lane to assume the next minimum threshold to broadcast. In general, the Congestion Control Model (CCM), result indicate that the lanes would follow this sequence: lane4; lane 3; lane 2; and lane 1; from the highest to the lowest in terms of traffic density. This means that, as the value of the $\lambda$ increases all the lane would also experience direct increases in each lane. 


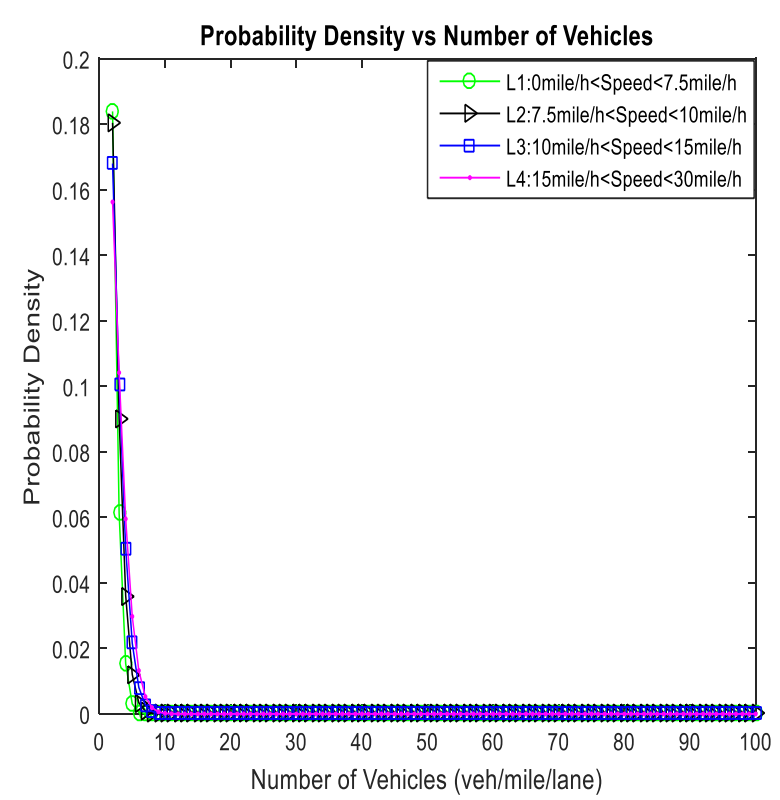

Figure 3. The effect of traffic flow when $\lambda=1$

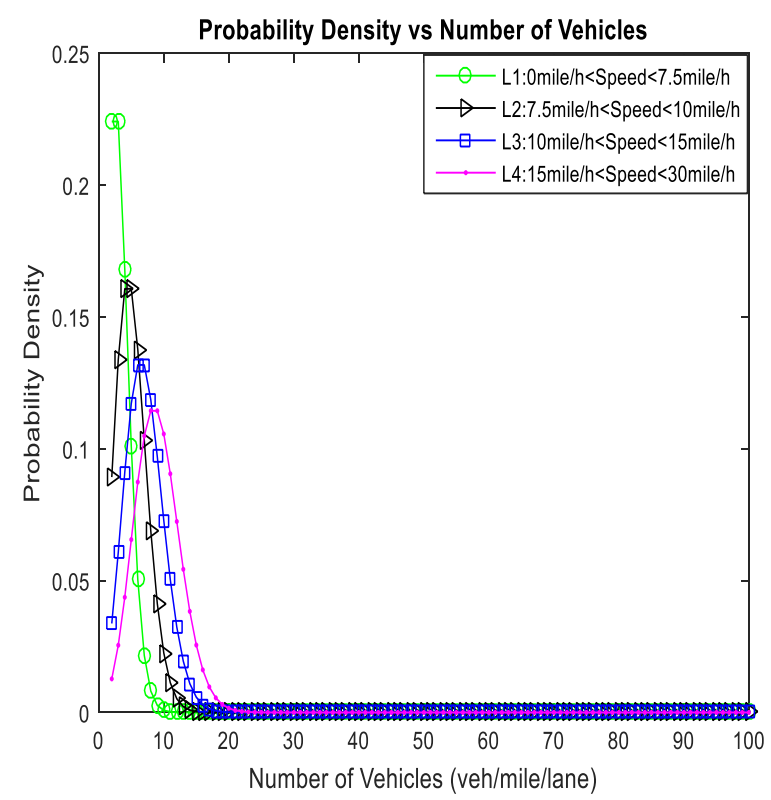

Figure 5. The effect of traffic flow when $\lambda=3$

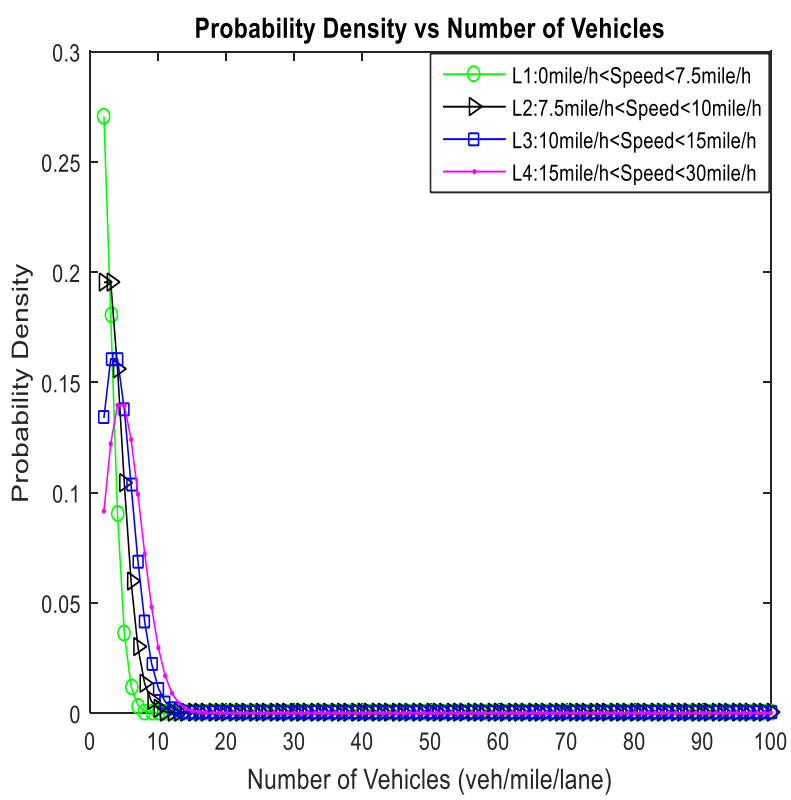

Figure 4. The effect of traffic flow when $\lambda=2$

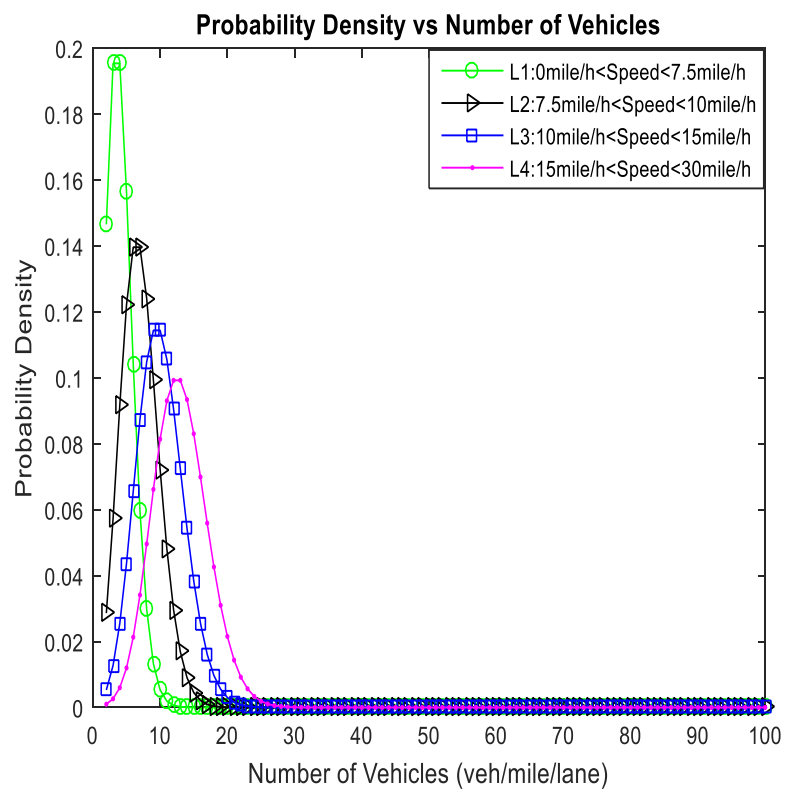

Figure 6. The effect of traffic flow when $\lambda=4$ 


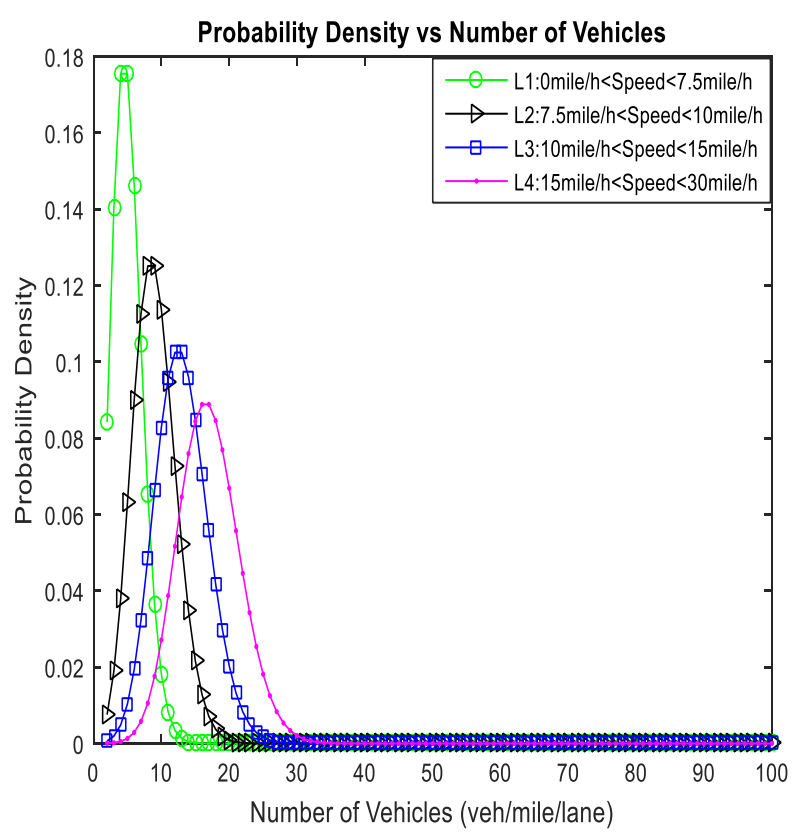

Figure 7. The effect of traffic flow when $\lambda=5$

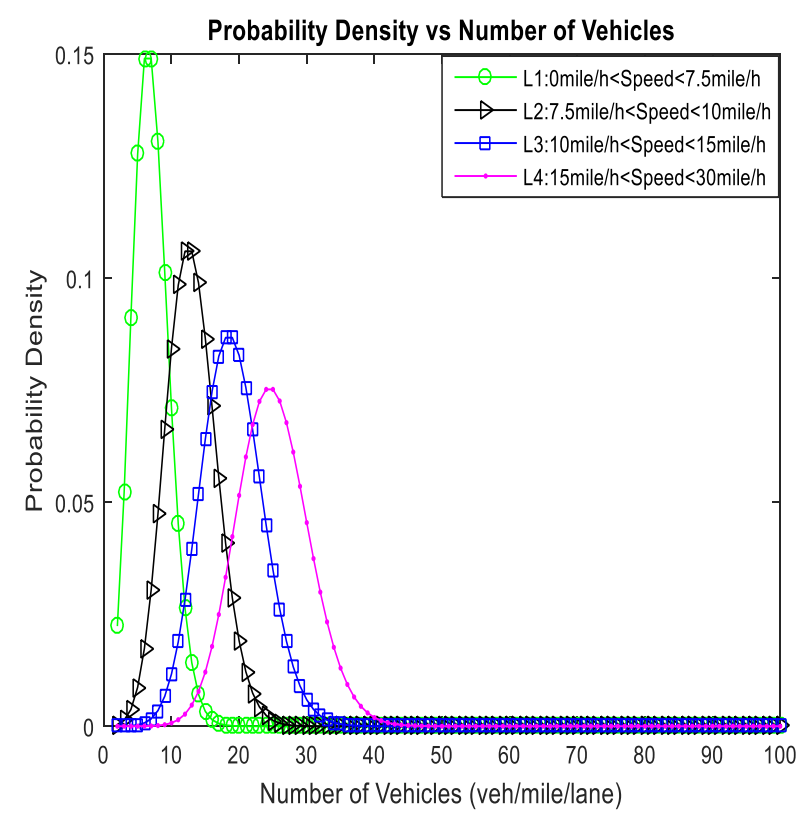

Figure 9. The effect of traffic flow when $\lambda=7$

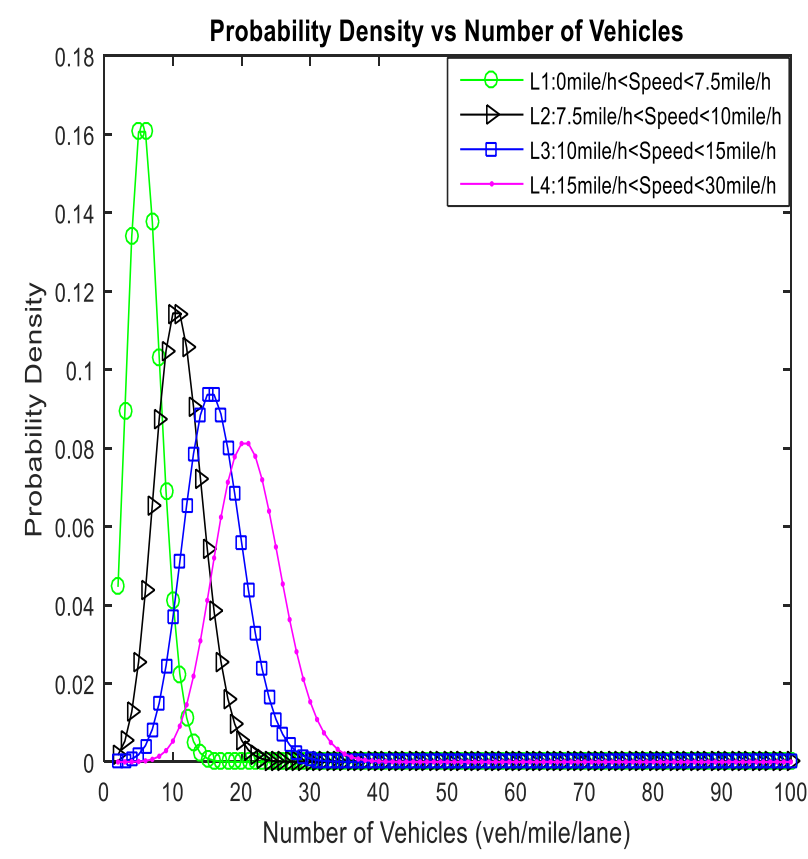

Figure 8. The effect of traffic flow when $\lambda=6$

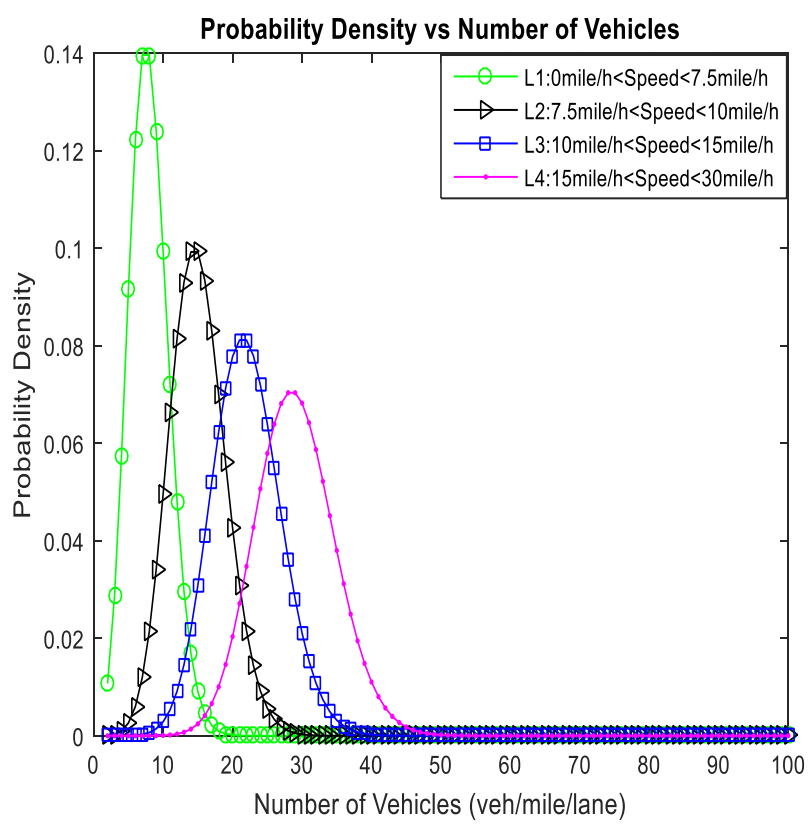

Figure 10. The effect of traffic flow when $\lambda=8$ 


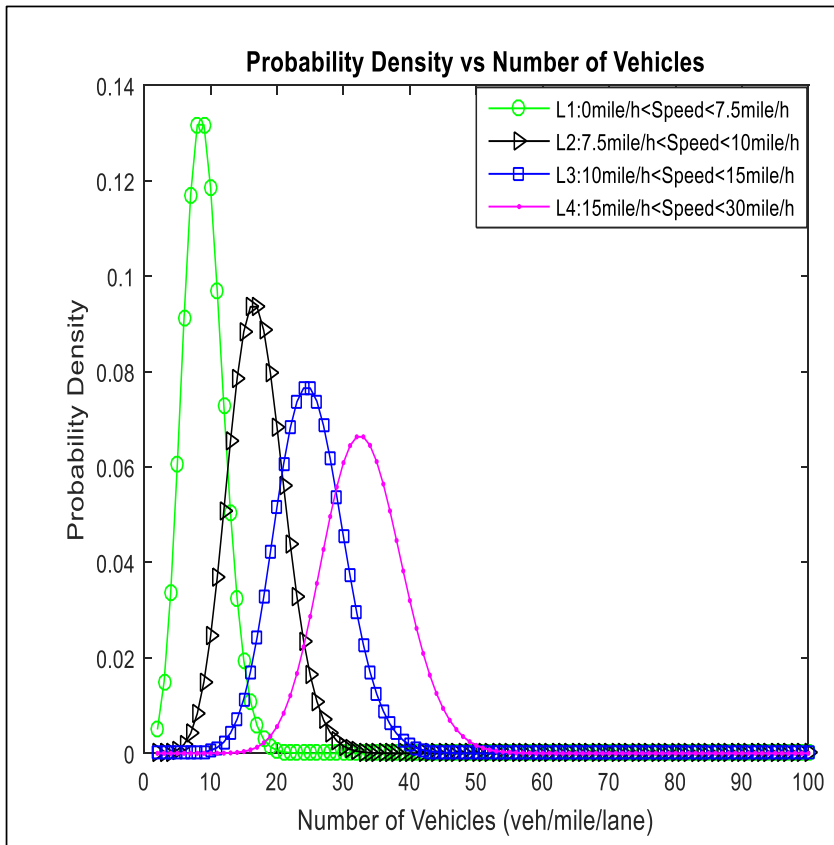

Figure 11. The effect of traffic flow when $\lambda=9$

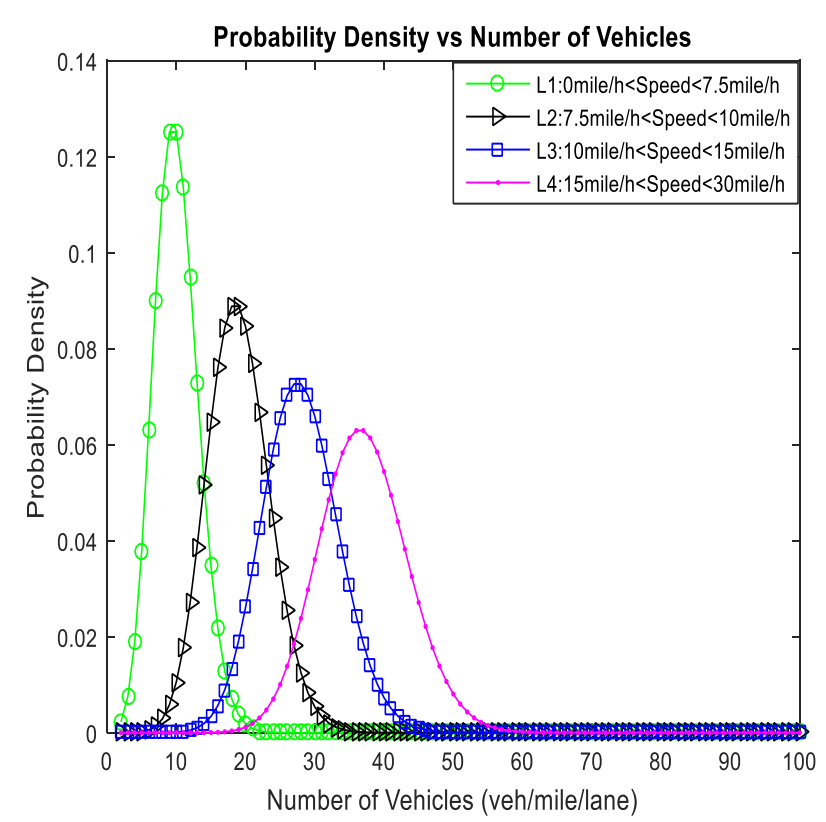

Figure 12. The effect of traffic flow when $\lambda=10$
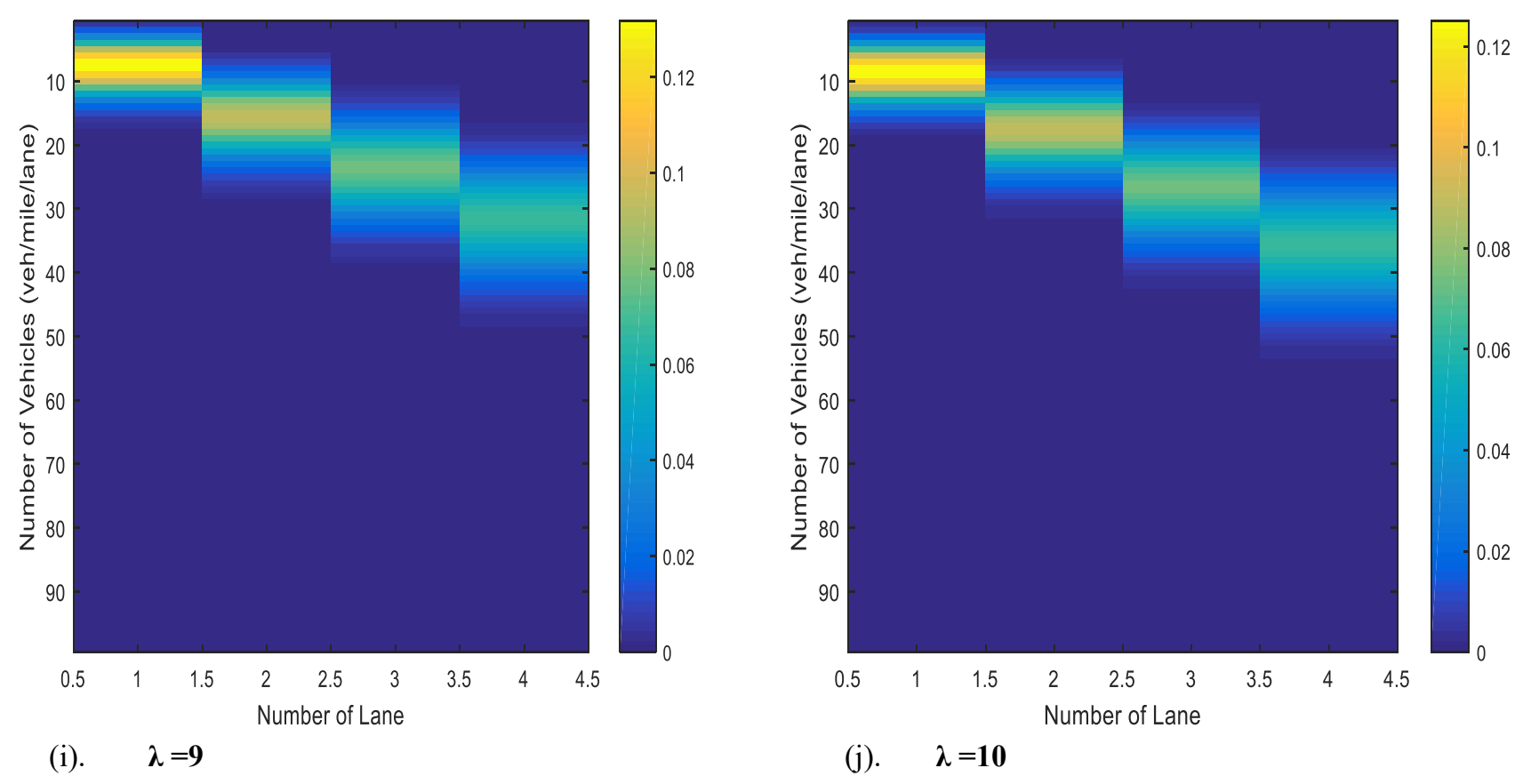


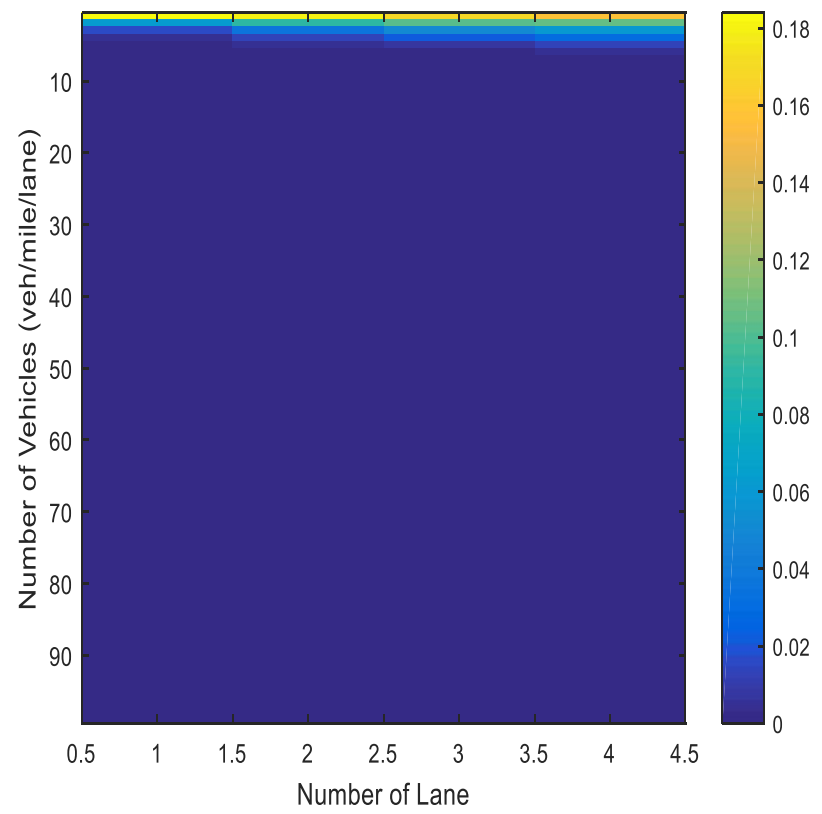

(a). $\lambda=1$

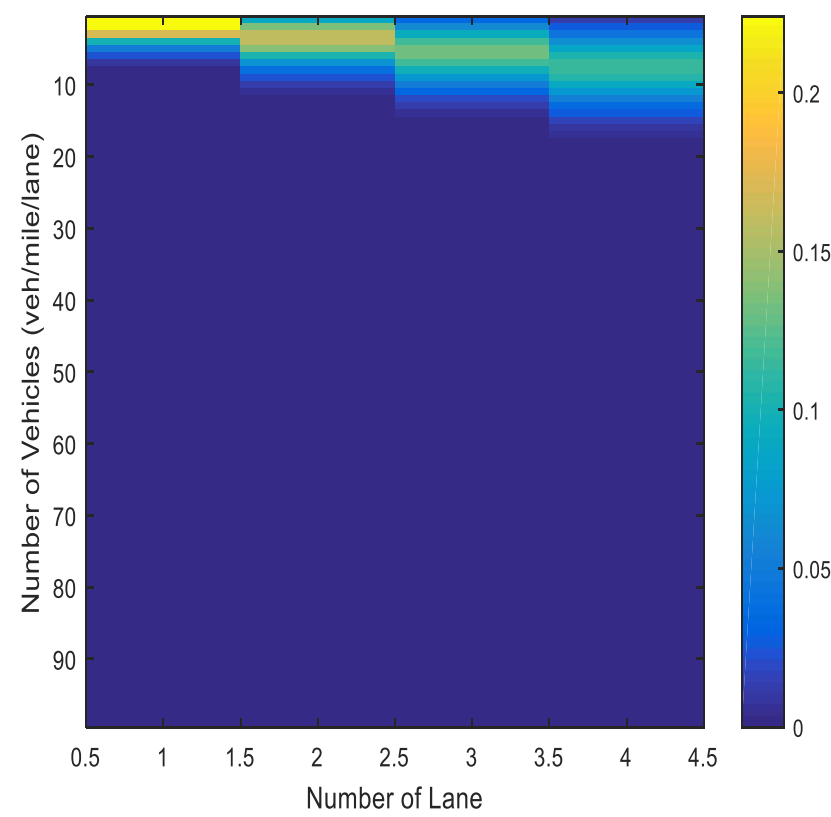

(c). $\lambda=\mathbf{3}$

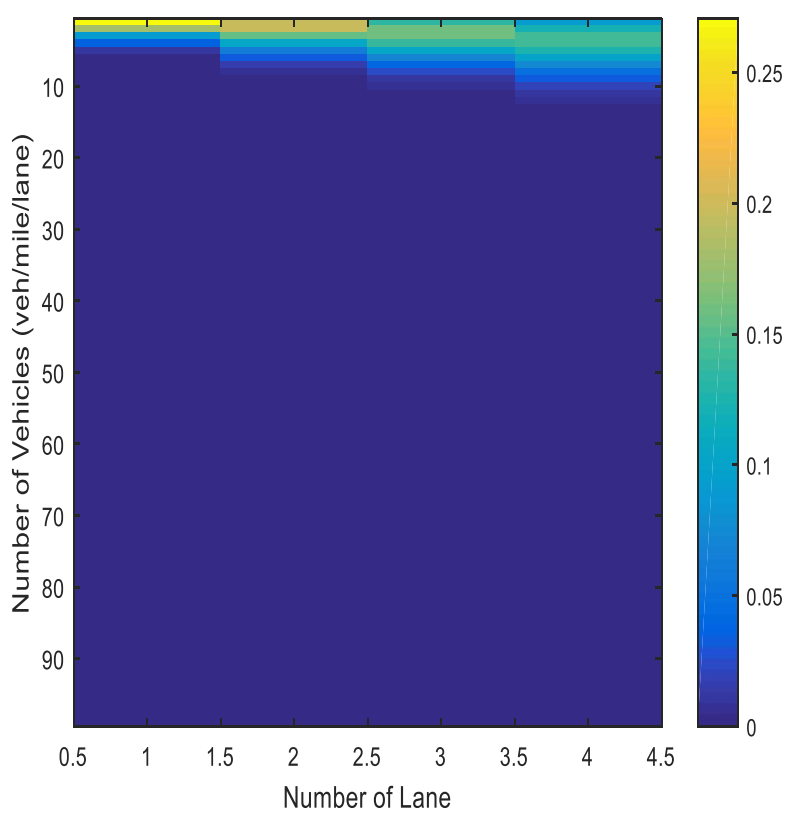

(b). $\lambda=\mathbf{2}$

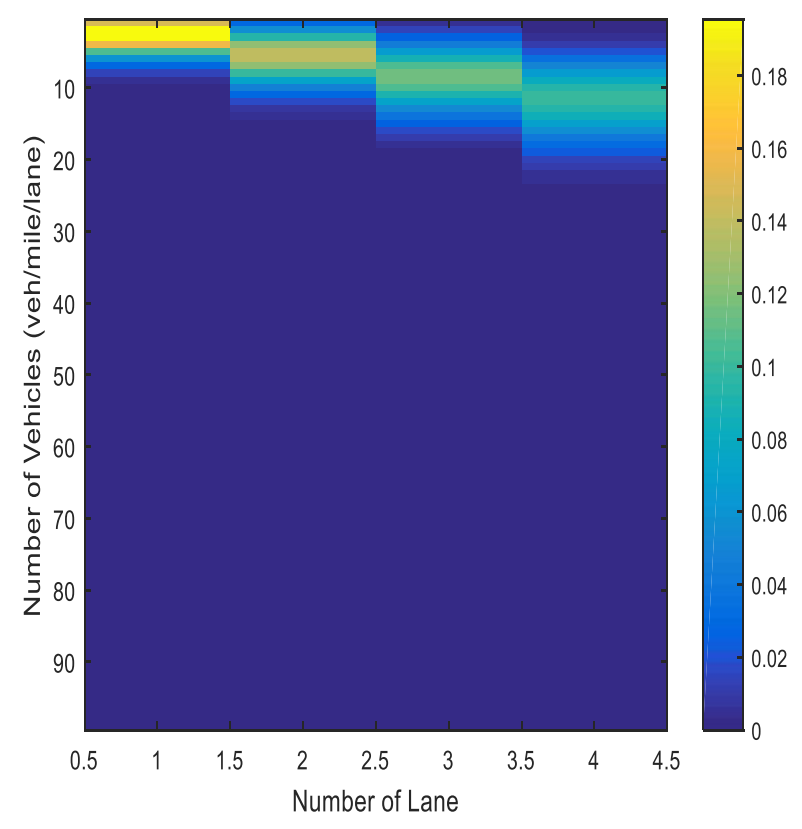

(d). $\quad \lambda=4$ 


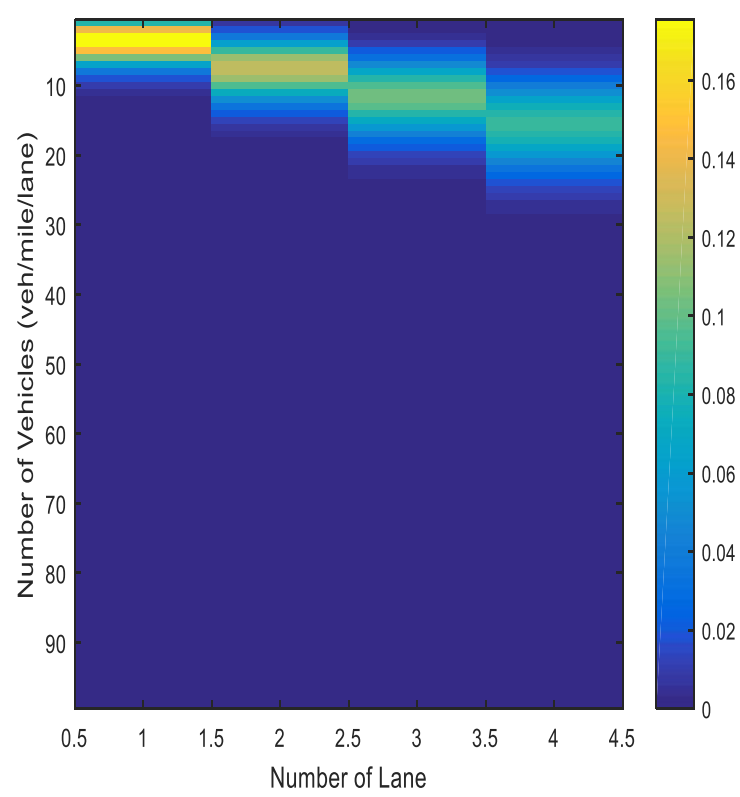

(e). $\quad \lambda=5$

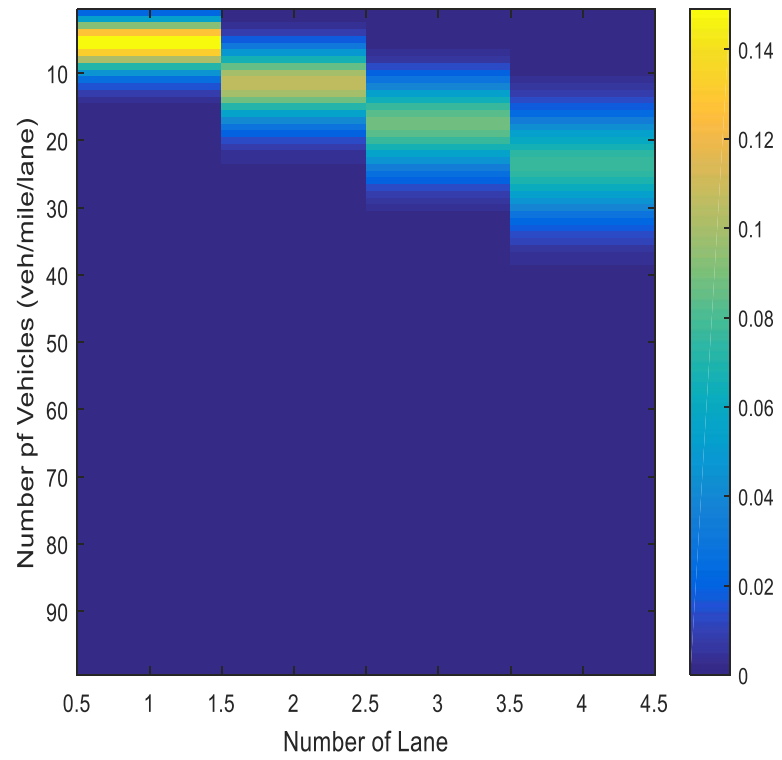

(g). $\quad \lambda=7$

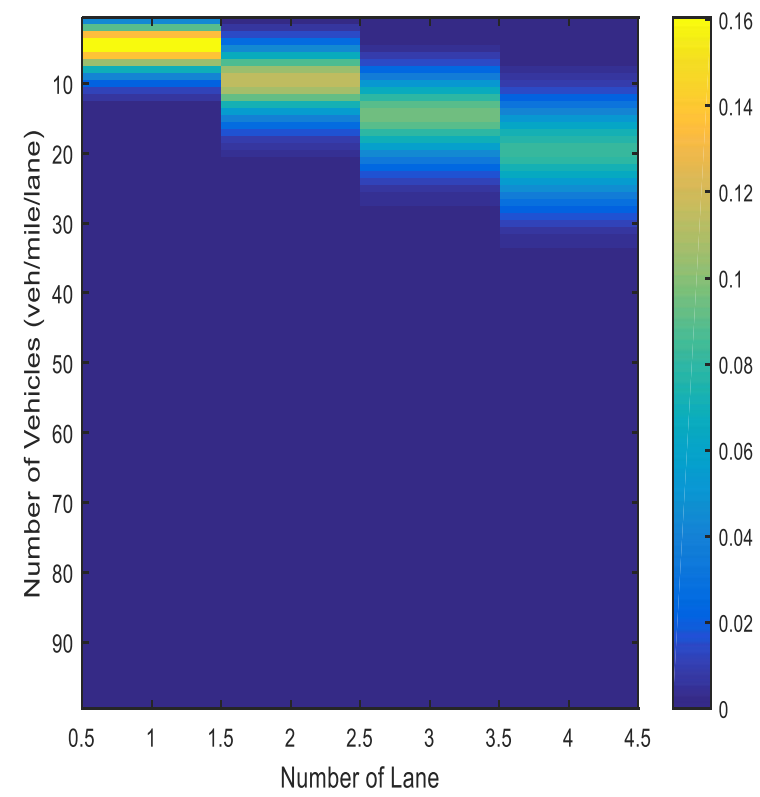

(f). $\lambda=6$

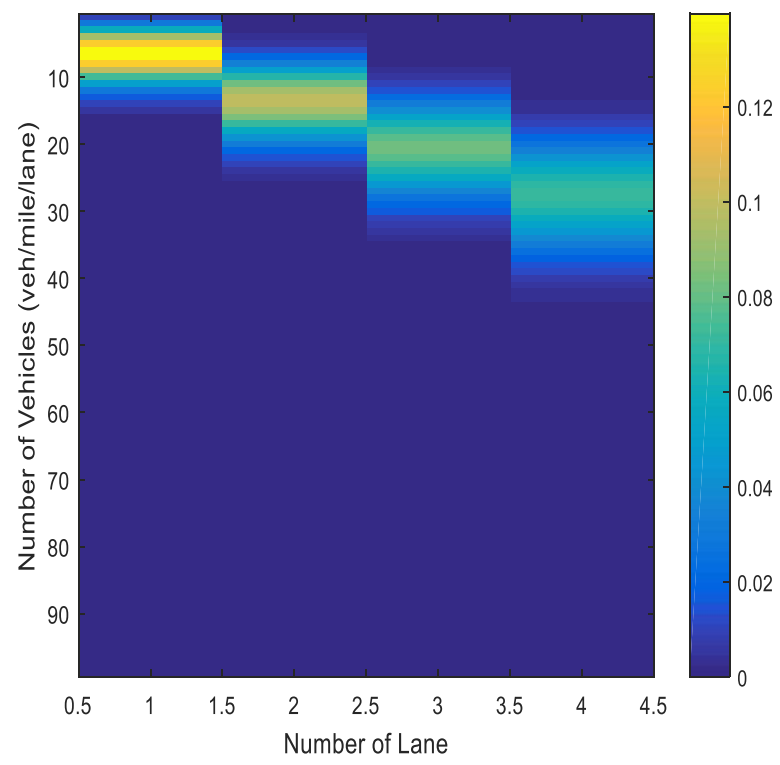

(h). $\lambda=\mathbf{8}$ 


\section{Conclusion and Future Work}

This study used traffic flow models (i.e. Civil Engineering) and concentrated on the macroscopic model sated in [45] and has presented a scalable solution to a congestion control in highly populated city environment. This proposed approach is a Congestion Control model designed for a four-lane road networks. The lanes are numbered from 1 to 4 and in either direction.

The model is able to indicate the exact lane with the highest vehicular density in sequence and that sequence is regarded as the highest congested lane and with the minimum threshold to receive broadcast messages follow by the next congested network with the minimum threshold to receive the next broadcast message and in that sequence while the vehicles move until the congestion is full controlled within the four-lane in both directions. The model is capable of eliminating message duplication and hence help reduce network overhead.

The simulation in MATLAB provides a practical implementation of V2V and V2R Communication where each lane shows the vehicle trajectories and the lane with the highest congestion coupled with the spatial variation in each successive lane. Therefore, vehicular movement is not entirely random but also sequential and depend largely on the drivers' perception and the interactions with other road users. This Congestion Control

\section{References}

[1] T. Nadeem, S. Dashtinezhad, C. Liao, and L. Iftode, "TrafficView: traffic data dissemination using car-to-car communication," ACM SIGMOBILE Mobile Computing and Communications Review, vol. 8, no. 3, pp. 6-19, 2004.

[2] A. Vinel, "3GPP LTE versus IEEE 802.11 p/WAVE: Which technology is able to support cooperative vehicular safety applications?," IEEE Wireless Communications Letters, vol. 1, no. 2, pp. 125-128, 2012.

[3] A. Balasubramanian, R. Mahajan, A. Venkataramani, B. N. Levine, and J. Zahorjan, "Interactive wifi connectivity for moving vehicles," ACM SIGCOMM Computer Communication Review, vol. 38, no. 4, pp. 427438, 2008.

[4] L. Wischhof, A. Ebner, and H. Rohling, "Information dissemination in self-organizing intervehicle networks," IEEE Transactions on intelligent transportation systems, vol. 6, no. 1, pp. 90-101, 2005.

[5] G. Korkmaz, E. Ekici, F. Özgüner, and Ü. Özgüner, "Urban multi-hop broadcast protocol for inter-vehicle communication systems," in Proceedings of the 1st ACM international workshop on Vehicular ad hoc networks, 2004, pp. 76-85: ACM.

[6] Z. D. Chen, H. Kung, and D. Vlah, "Ad hoc relay wireless networks over moving vehicles on highways," in Proceedings of the 2nd ACM
Model (CCM) would help in designing vehicular traffic flow and there is no need increasing the value of $\lambda$ beyond this range $1 \leq \lambda$ $\leq 12$ in congested networks.

This study provides fast, reliable and scalable model to congestion control. Although, there exist many interventions to provide a lasting solution to congestion problem in VANETs, this proposed Congestion Control Model (CCM) provide an alternative methodology and framework for the research community to develop lasting protocol for safety alert message dissemination in VANETs.

international symposium on Mobile ad hoc networking \& computing, 2001, pp. 247-250: ACM.

[7] H. Sasaki, H. Iwasaki, and T. Suda, "Simulation of information propagation for vehicles in physical communication network models," in Applications and the Internet Workshops, 2004. SAINT 2004 Workshops. 2004 International Symposium on, 2004, pp. 408-414: IEEE.

[8] J. Tian, L. Han, and K. Rothermel, "Spatially aware packet routing for mobile ad hoc intervehicle radio networks," in Intelligent Transportation Systems, 2003. Proceedings. 2003 IEEE, 2003, vol. 2, pp. 1546-1551: IEEE.

[9] L. Briesemeister, L. Schafers, and G. Hommel, "Disseminating messages among highly mobile hosts based on inter-vehicle communication," in Intelligent Vehicles Symposium, 2000. IV 2000. Proceedings of the IEEE, 2000, pp. 522-527: IEEE.

[10] M. Thomas, E. Peytchev, and D. Al-Dabass, "Auto-sensing and distribution of traffic information in vehicular ad hoc networks," in Proceedings of the United Kingdom Simulation Society Conference, 2004, pp. 124-128.

[11] S. Al-Sultan, M. M. Al-Doori, A. H. Al-Bayatti, and H. Zedan, "A comprehensive survey on vehicular ad hoc network," Journal of network and computer applications, vol. 37, pp. 380-392, 2014.

[12] F. J. Martinez, C. K. Toh, J. C. Cano, C. T. Calafate, and P. Manzoni, "A survey and comparative study of simulators for vehicular ad hoc networks (VANETs)," Wireless Communications and Mobile Computing, vol. 11, no. 7, pp. 813-828, 2011. 
[13] G. Korkmaz, E. Ekici, and F. Özgüner, "Supporting real-time traffic in multihop vehicleto-infrastructure networks," Transportation Research Part C: Emerging Technologies, vol. 18, no. 3, pp. 376-392, 2010.

[14] S. Ukkusuri and L. Du, "Geometric connectivity of vehicular ad hoc networks: Analytical characterization," Transportation Research Part C: Emerging Technologies, vol. 16, no. 5, pp. 615-634, 2008.

[15] S. Ghandeharizade, S. Kapadia, and B. Krishnamachari, "PAVAN: a policy framework for content availabilty in vehicular ad-hoc networks," in Proceedings of the 1st ACM international workshop on Vehicular ad hoc networks, 2004, pp. 57-65: ACM.

[16] M. Ferreira, R. Fernandes, H. Conceição, W. Viriyasitavat, and O. K. Tonguz, "Self-organized traffic control," in Proceedings of the seventh ACM international workshop on VehiculAr InterNETworking, 2010, pp. 85-90: ACM.

[17] O. Tonguz, N. Wisitpongphan, F. Bait, P. Mudaliget, and V. Sadekart, "Broadcasting in VANET," in 2007 mobile networking for vehicular environments, 2007, pp. 7-12: IEEE.

[18] J. Härri, F. Filali, C. Bonnet, and M. Fiore, "VanetMobiSim: generating realistic mobility patterns for VANETs," in Proceedings of the 3rd international workshop on Vehicular ad hoc networks, 2006, pp. 96-97: ACM.

[19] M. Raya, A. Aziz, and J.-P. Hubaux, "Efficient secure aggregation in VANETs," in Proceedings of the 3rd international workshop on Vehicular ad hoc networks, 2006, pp. 67-75: ACM.

[20] B. Greenshields, W. Channing, and H. Miller, "A study of traffic capacity," in Highway research board proceedings, 1935, vol. 1935: National Research Council (USA), Highway Research Board.

[21] S. A. Boxill and L. Yu, "An evaluation of traffic simulation models for supporting its," Houston, TX: Development Centre for Transportation Training and Research, Texas Southern University, 2000.

[22] J. Tian, J. Hahner, C. Becker, I. Stepanov, and K. Rothermel, "Graph-based mobility model for mobile ad hoc network simulation," in Simulation Symposium, 2002. Proceedings. 35th Annual, 2002, pp. 337-344: IEEE.

[23] E. C. Eze, S.-J. Zhang, E.-J. Liu, and J. C. Eze, "Advances in vehicular ad-hoc networks (VANETs): Challenges and road-map for future development," International Journal of Automation and Computing, vol. 13, no. 1, pp. 118, 2016.

[24] H. Hartenstein and L. Laberteaux, "A tutorial survey on vehicular ad hoc networks," IEEE Communications magazine, vol. 46, no. 6, 2008.
[25] S. Zeadally, R. Hunt, Y.-S. Chen, A. Irwin, and A. Hassan, "Vehicular ad hoc networks (VANETS): status, results, and challenges," Telecommunication Systems, vol. 50, no. 4, pp. 217-241, 2012.

[26] V. Naumov, R. Baumann, and T. Gross, "An evaluation of inter-vehicle ad hoc networks based on realistic vehicular traces," in Proceedings of the 7th ACM international symposium on Mobile ad hoc networking and computing, 2006, pp. 108119: ACM.

[27] M. Rudack, M. Meincke, K. Jobmann, and M. Lott, "On traffic dynamical aspects of inter vehicle communications (IVC)," in Vehicular Technology Conference, 2003. VTC 2003-Fall. 2003 IEEE 58th, 2003, vol. 5, pp. 3368-3372: IEEE.

[28] M. Rudack, M. Meincke, and M. Lott, "On the dynamics of ad hoc networks for inter vehicle communications (IVC)," in proc. ICWN, 2002, vol. 2.

[29] J. Harri, F. Filali, and C. Bonnet, "Mobility models for vehicular ad hoc networks: a survey and taxonomy," IEEE Communications Surveys \& Tutorials, vol. 11, no. 4, 2009.

[30] R. Weil, J. Wootton, and A. Garcia-Ortiz, "Traffic incident detection: Sensors and algorithms," Mathematical and computer modelling, vol. 27, no. 9-11, pp. 257-291, 1998.

[31] A. Ebner and H. Rohling, "A self-organized radio network for automotive applications," in Conference Proceedings ITS 2001, 8th World Congress on Intelligent Transportation Systems, 2001.

[32] M. Adachi, Y. Morita, K. Fujimura, Y. Takatori, and T. Hasegawa, "On an autonomous cruising traffic flow simulator including inter-vehicle and road-to-vehicle communication networks," in Intelligent Transportation Systems, 2002. Proceedings. The IEEE 5th International Conference on, 2002, pp. 645-650: IEEE.

[33] H. Füßler, M. Mauve, H. Hartenstein, M. Käsemann, and D. Vollmer, "A comparison of routing strategies for vehicular ad hoc networks," Technical reports, vol. 2, 2002.

[34] N. R. C. T. R. B. C. f. G. o. Setting and E. S. Limits, Managing speed: review of current practice for setting and enforcing speed limits. Transportation Research Board, 1998.

[35] J. Milazzo II, N. Rouphail, J. Hummer, and D. Allen, "Quality of service for interrupted-flow pedestrian facilities in highway capacity manual 2000," Transportation Research Record: Journal of the Transportation Research Board, no. 1678, pp. 25-31, 1999.

[36] M. Haglund, "Speed Choice: The Driver, the Road and Speed Limits," Acta Universitatis Upsaliensis, 2001. 
[37] S. L. Johnson and N. Pawar, "Cost-benefit evaluation of large truck-automobile speed limit differentials on rural interstate highways," MackBlackwell National Rural Transportation Study Center, University of Arkansas2005.

[38] R. J. Cowan, "Useful headway models," Transportation Research, vol. 9, no. 6, pp. 371375, 1975.

[39] J. Duan, Z. Li, and G. Salvendy, "Risk illusions in car following: Is a smaller headway always perceived as more dangerous?," Safety science, vol. 53, pp. 25-33, 2013.

[40] G. Naus, R. Van Den Bleek, J. Ploeg, B. Scheepers, R. van de Molengraft, and $\mathrm{M}$. Steinbuch, "Explicit MPC design and performance evaluation of an ACC Stop-\&-Go," in American Control Conference, 2008, 2008, pp. 224-229: IEEE.

[41] V. Kanagaraj, G. Asaithambi, C. N. Kumar, K. K. Srinivasan, and R. Sivanandan, "Evaluation of different vehicle following models under mixed traffic conditions," Procedia-Social and Behavioral Sciences, vol. 104, pp. 390-401, 2013.

[42] R. Mangharam, "Real-time embedded wireless networks: Algorithms and experiences," Carnegie Mellon University, 2008.

[43] D. R. Choffnes and F. E. Bustamante, "An integrated mobility and traffic model for vehicular wireless networks," in Proceedings of the 2nd ACM international workshop on Vehicular ad hoc networks, 2005, pp. 69-78: ACM.

[44] C. Lochert, H. Hartenstein, J. Tian, H. Fussler, D. Hermann, and M. Mauve, "A routing strategy for vehicular ad hoc networks in city environments," in Intelligent Vehicles Symposium, 2003. Proceedings. IEEE, 2003, pp. 156-161: IEEE.
[45] D. Adolf, "May. Traffic flow fundamentals," University of California, Berkeley, 1990.

[46] H. Yang and S. Yagar, "Traffic assignment and traffic control in general freeway-arterial corridor systems," Transportation Research Part B: Methodological, vol. 28, no. 6, pp. 463-486, 1994.

[47] K. Nagel and H. J. Herrmann, "Deterministic models for traffic jams," Physica A: Statistical Mechanics and its Applications, vol. 199, no. 2, pp. 254-269, 1993.

[48] M. Rickert, K. Nagel, M. Schreckenberg, and A. Latour, "Two lane traffic simulations using cellular automata," Physica A: Statistical Mechanics and its Applications, vol. 231, no. 4, pp. 534-550, 1996.

[49] A. D. May, Traffic flow fundamentals. 1990.

[50] G. Kesidis, A. Singh, D. Cheung, and W. Kwok, "Feasibility of fluid event-driven simulation for ATM networks," in Global Telecommunications Conference, 1996. GLOBECOM'96.'Communications: The Key to Global Prosperity, 1996, vol. 3, pp. 2013-2017: IEEE.

[51] A. Donev, S. Torquato, and F. H. Stillinger, "Neighbor list collision-driven molecular dynamics simulation for nonspherical hard particles. I. Algorithmic details," Journal of computational physics, vol. 202, no. 2, pp. 737764, 2005.

[52] B. D. Lubachevsky, "Efficient distributed eventdriven simulations of multiple-loop networks," Communications of the ACM, vol. 32, no. 1, pp. 111-123, 1989. 